American Journal of Applied Sciences 5 (9): 1233-1238, 2008

ISSN 1546-9239

(C) 2008 Science Publications

\title{
Aerodynamic Noise Prediction Using stochastic Turbulence Modeling
}

\author{
Arash Ahmadzadegan and Mehran Tadjfar \\ Center of Excellence in Computational Aerospace Engineering (AeroExcel) \\ Aerospace Engineering Department, Amirkabir University of Technology, Tehran, Iran
}

\begin{abstract}
Amongst many approaches to determine the sound propagated from turbulent flows, hybrid methods, in which the turbulent noise source field is computed or modeled separately from the far field calculation, are frequently used. For basic estimation of sound propagation, less computationally intensive methods can be developed using stochastic models of the turbulent fluctuations (turbulent noise source field). A simple and easy to use stochastic model for generating turbulent velocity fluctuations called continuous filter white noise (CFWN) model was used. This method based on the use of classical Langevian-equation to model the details of fluctuating field superimposed on averaged computed quantities. The resulting sound field due to the generated unsteady flow field was evaluated using Lighthill's acoustic analogy. Volume integral method used for evaluating the acoustic analogy. This formulation presents an advantage, as it confers the possibility to determine separately the contribution of the different integral terms and also integration regions to the radiated acoustic pressure. Our results validated by comparing the directivity and the overall sound pressure level (OSPL) magnitudes with the available experimental results. Numerical results showed reasonable agreement with the experiments, both in maximum directivity and magnitude of the OSPL. This method presents a very suitable tool for the noise calculation of different engineering problems in early stages of the design process where rough estimates using cheaper methods are needed for different geometries.
\end{abstract}

Key words: Computational Aeroacoustics, Lighthill's Volume Integral, Hybrid Method, Jet Acoustics

\section{INTRODUCTION}

One of the major contributors to the overall aircraft's noise is its propulsive jet. In order to design quieter aircrafts, noise reduction in jets has become a major area of jet research ${ }^{[1]}$. This is a difficult task to be done because of the noticeable inefficiency of turbulence as an acoustic source. When there is no solid surface in the flow field, quadrupole acoustic sources formed by the turbulent Reynolds stresses are responsible for generating most of the sound ${ }^{[2]}$. Three hybrid methods may be used in computational aeroacoustics to study compressible jet flows. Each method has its own particular way for computing the near field turbulent flow and far field noise data ${ }^{[3]}$. First approach relies on direct numerical simulation (DNS) in which near field is computed by solving the full compressible Navier-Stokes equations. However the practical application of DNS is limited to low Reynolds numbers with simple geometries. Second approach uses the mean turbulent flow field computed using some turbulence modeling method combined with statistical source representation for the noise. In the third approach, the turbulent mean flow is computed as in the second method, but the details of the turbulent fluctuation field are regenerated by stochastic or random-walk models. Then Lighthill's analogy or Kirchhoff integral ${ }^{[4]}$ is used to estimate the noise in the far field.

In all of the above methods, computing the near field has to be done first. Stochastic or random-walk models have proved to be a successful and flexible tool for simulating turbulent fluctuations in high-Reynoldsnumber turbulent flows. They can take account of inhomogeneities, unsteadiness or non-Gaussian distributions in the flow. They can also be used for complex flows ${ }^{[5]}$. Statistical methods are also used for subgrid scale modeling in LES simulations ${ }^{[6]}$. In this approach large eddies are solved numerically and small eddies are modeled stochastically. More thorough descriptions of various computational aeroacoustic methods with more emphasis on the hybrid methods can be found in ${ }^{[7,8]}$.

Here we used the volume integration methods for the far field noise prediction instead of the more common surface integral methods. This type of

Corresponding Author: Prof. Mehran Tadjfar, Amirkabir University of Technology, Tehran 15875-4413, Iran 
acoustic post treatment renders the CFD calculation less computationally intensive. The volume integral approach also seems advantageous for it allows a detailed physical examination of the noise creation process, through the differentiation of the source types (entropy, shear,...), and through the analysis of the spatial distribution of noise sources. ${ }^{[9]}$

In this paper, turbulent mean flow of a two dimensional, compressible, cold-jet at mach 0.56 is computed using RANS with 2 equation $k-\varepsilon$ RNG model, then the mean-flow quantities are exported for use in the stochastic turbulence generation code to simulate the fluctuating velocities and finally computation of the far field noise is done using the Lighthill's volume integration method.

\section{MATERIALS AND METHODS}

Characteristics of the Two-Dimensional Jets Technical Work Preparation:

We considered a free cold-jet configuration for applying our method because most of the references and available data in the literature are regarding this problem. In a free cold-jet configuration due to very large velocity differences at the surface of discontinuity, large eddies are formed that cause intense lateral mixing. We know that in the zone of establishment of the jet, there is a core region that has constant velocity and very little turbulence. After the zone of establishment, diffusion of the momentum of ambient fluid reaches the centerline of the jet and the mean velocity on the symmetry line starts to decrease downstream thereafter. Figure 1 shows these properties of a free jet.

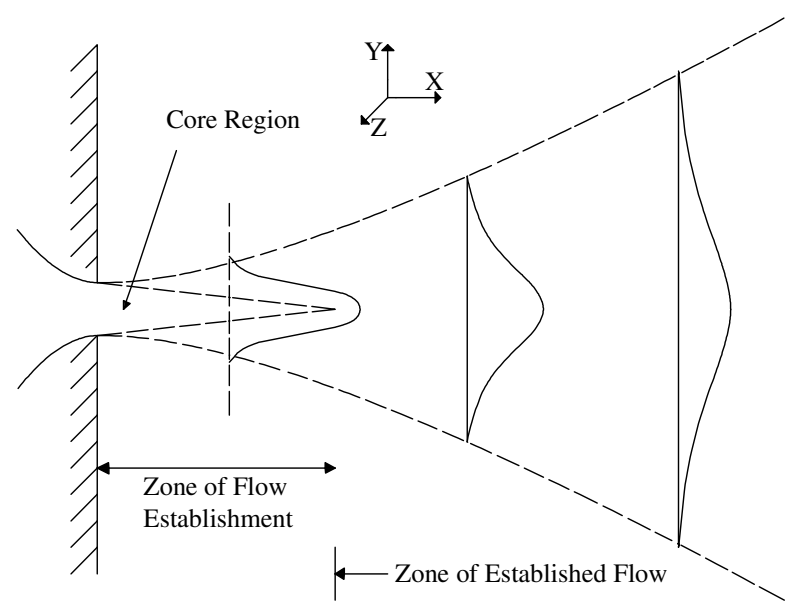

Fig. 1: 2D free jet

The geometry and the computational domain of the two dimensional jet used for calculating the mean turbulent flow is presented below in Fig. 2.

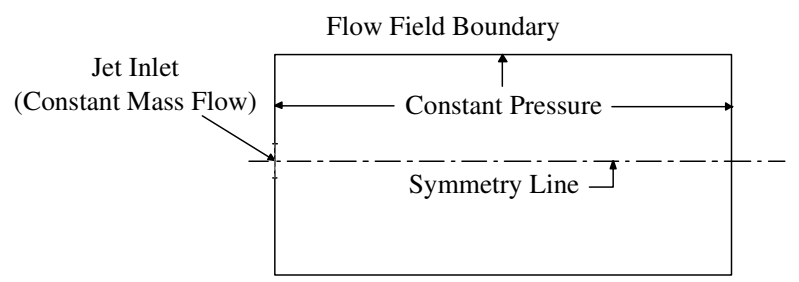

Fig. 2: Geometry of the computational domain and its boundary conditions

The lowest Reynolds number used in this study is chosen to be at least 200000 . Hence, it is much higher than the critical Reynolds number of a free jet.

As our stochastic method (which will be discussed more in the next chapter) needs the kinetic energy of turbulence and also the rate of dissipation of kinetic energy of turbulence in each grid point, here we choose to solve the RANS equations with the 2 equation $k-\varepsilon$ method for its closure. A simple 2 equation $k-\varepsilon$ RANS solver code is used on a structured grid for this purpose.

Because of the fact that the mean turbulent quantities are symmetrically distributed, only half of the flow field above the symmetry line was considered for computing the mean quantities of the turbulent flow. All boundaries have constant pressure imposed as boundary condition.

\section{Description of the Stochastic Model:}

The turbulence fluctuations are random-like functions of space and time. In this study the continuous filter white noise (CFWN) model ${ }^{[10]}$, which is based on the classical Langevian-equation ${ }^{[5]}$ was used to simulate the instantaneous fluctuating velocity of the flow field.

$$
\begin{gathered}
\frac{d u_{i}}{d t}=-\frac{u_{i}-\bar{u}_{i}}{T_{I}}+\left(\frac{2 \overline{u_{i}^{\prime 2}}}{T_{I}}\right)^{1 / 2} \zeta_{i}(t) \\
\text { Where, } \overline{u_{i}^{\prime 2}} \text { is the mean-square of the } i \text { th }
\end{gathered}
$$

fluctuating velocity, and the summation convention on underlined indices is avoided. $T_{I}$ is the Lagrangian integral time $T_{I}=0.30 \mathrm{k} / \varepsilon . \quad \zeta_{i}(t)$ is a Gaussian vector white noise random function with spectral intensity $S_{i j}^{n}=\delta_{i j} / \pi$. This in the numerical method is computed as $G_{i} / \sqrt{\Delta t}$. Where $G_{i}$ is a zero-mean unit variance independent Gaussian random number and has to be computed at every time step, $\Delta t$, in the entire time domain.

Equation 1 has to be solved for each direction of the flow field independently to obtain the velocity fluctuations in that direction. The numerical data needed for solving Equation 1 at each point of the flow field are the mean velocities, kinetic energy of turbulence $(k)$, rate of dissipation of kinetic energy of turbulence $(\varepsilon)$, (All computed in the RANS solver), and 
the Gaussian random numbers $G_{i}$, which is generated using the polar form of the Box-Muller transformation.

This is a fast and robust method to generate Gaussian random numbers ${ }^{[11]}$. Here, Eq. 1 is solved analytically and only the integration in the analytical solution was computed numerically. This way less computational error is introduced.

Since different equations are solved for each dimension, the generated turbulence field is not necessarily isotropic. Also note that this equation takes into account the intensity of local turbulence at each point ala the use of kinetic energy and dissipation rate in the formulation. This technique has some advantages compared to other techniques. It provides correct turbulent intensities and accounts for the proper time scale of turbulence. More importantly the model leads to the correct magnitude of turbulent diffusivity at each fluid point particle ${ }^{[10]}$.

\section{Evaluation of the Far Field Noise:}

In order to evaluate the far field noise emitted from the turbulent velocity distribution, we use the volume integration as prescribed by Lighthill's analogy ${ }^{[2]}$ :

$$
\rho-\rho_{0}=\frac{1}{4 \pi a_{0}^{2}} \frac{\partial^{2}}{\partial x_{i} \partial x_{j}} \int T_{i j}\left(\bar{y}, t-\frac{|\bar{x}-\bar{y}|}{a_{0}}\right) \frac{d \bar{y}}{|\bar{x}-\bar{y}|}
$$

Where $T_{i j}$ is the Lighthill's quadrupole source and in most cases can be replaced by $\rho u_{i} u_{j}$. Note that $T_{i j}$ is calculated at the retarded time, which is the time needed for the sound waves to travel the distance between source and observer positions. Here, all the discritizations are done using 4th order finite difference schemes ${ }^{[12]}$.

Computing the sound propagated from a turbulent flow region with the aid of volume integral methods has its own cons and pros. If the use of a surface integral method avoids computing 3D integrals with double space or time derivatives, this approach nevertheless comes with certain difficulties. The acoustic pressure has to be calculated using CFD up to a control surface situated in the uniform flow, which imposes a fine mesh over a relatively extended domain. The use of volume integral methods for acoustics could alleviate this constraint since the pressure fronts don't have to be propagated up to the control surface. Furthermore, only the dominant noise production sources have to be finely captured.

The main drawback of our formulation, which uses double space derivative outside of the integral, is the increase of the computational cost of the acoustic computation. Actually since the space derivation takes place at the observer location, three integrals have to be computed for 24 points around the observer location.
This formulation confers the possibility to determine separately the contribution of the different integral terms and also spatial distribution of noise sources to the radiated pressure.

Figure 3 presents a schematic of the far-field and the computational flow region. The overall sound pressure level, OSPL, of the sound at far field is computed along the perimeter of a half circle with the radius $\bar{X}$ (position vector).

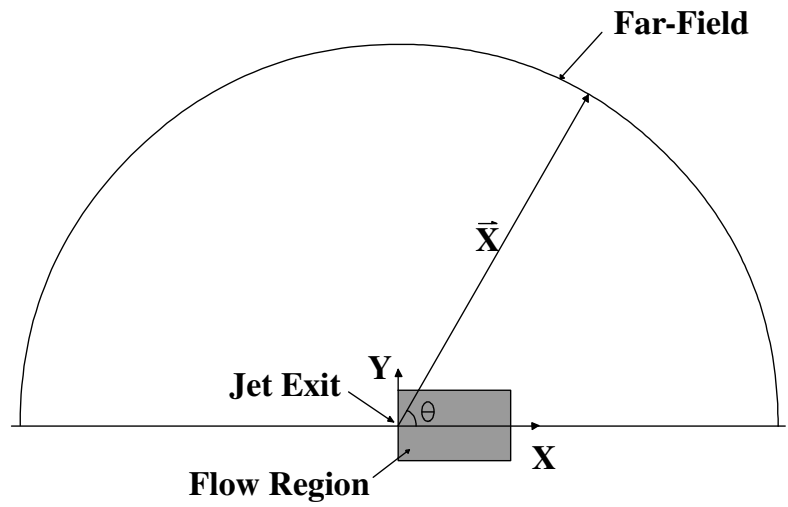

Fig. 3: Schematic of the jet geometry and far-field region

\section{RESULTS AND DISCUSSION}

\section{Evaluation of the Mean Flow Properties:}

To check the accuracy of our RANS numerical results, the mean velocity on the symmetry line of the jet is compared with the experimental data. The experimental profile suggested by Zijnen ${ }^{[13]}$ is rewritten below:

$\frac{\bar{u}_{m}}{U_{0}}=\frac{3.50}{\sqrt{x / b_{0}}}$

Where $b_{0}$ is the half of jet exit nozzle and $U_{0}$ is the jet velocity at the nozzle exit. In this study $U_{0}=190 \mathrm{~m} / \mathrm{s}$ and $b_{0}=0.0005 \mathrm{~m}$. The above experimental relation is only valid in the fully developed region of the jet flow.

In contrast, the results from RANS code are valid everywhere in the flow field. Hence, our numerical results are valid even in the potential core region of the jet. So comparison with experimental data can be done only in the fully developed region of the jet far from the nozzle exit. As shown in Fig. 4, the computed mean velocity on the symmetry line lies on the experimental data in the fully developed region of the jet (as it would have been expected). 


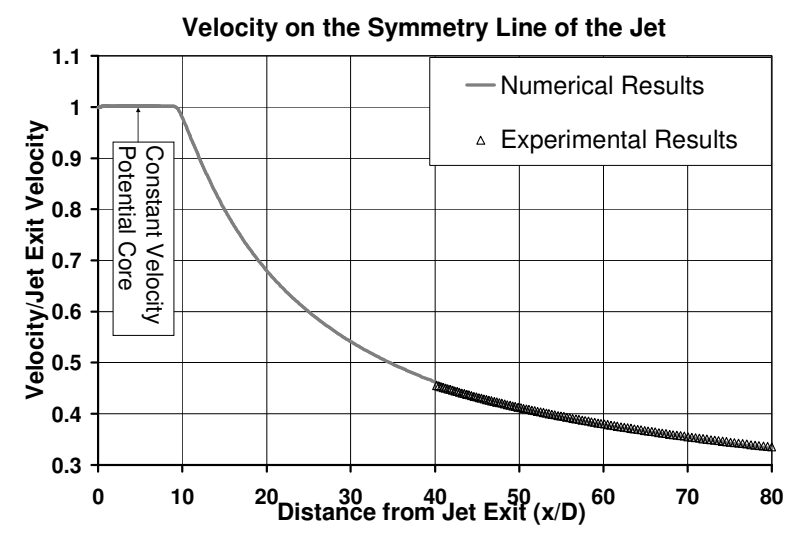

Fig. 4: Comparison of numerical with experimental velocities ${ }^{[4]}$ on the symmetry line

Another test that can be used to check the validity of the numerical results is the mean velocity profile along the lines normal to the symmetry line. Experimental data fit for velocity profiles from Zijnen ${ }^{[13]}$ is given below: (Where $a_{0}$ can vary from 70.7 to 75.0 )

$\frac{\bar{u}}{\bar{u}_{m}}=e^{-a_{0}\left(\frac{y}{x}\right)}$

We can also compare with the theoretical calculations of Görtler ${ }^{[14]}$ :

$$
\frac{\bar{u}}{\bar{u}_{m}}=1-\tanh ^{2} \xi \quad ; \quad \xi=\frac{\sigma y}{x}
$$

Experimental investigations have reported the value of $\sigma$ to be around $7.67^{[14]}$.

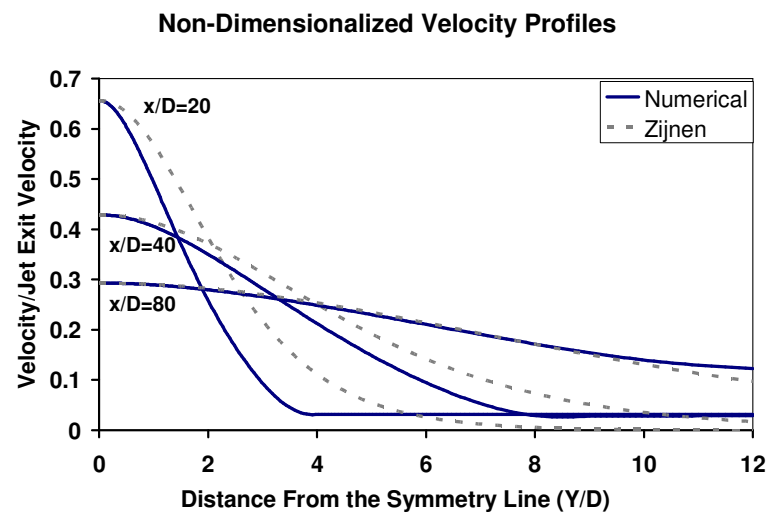

Fig. 5: Comparison of the computed jet velocity profile with the experimental data ${ }^{[13]}$.

In Fig. 5, the comparison between the numerical results is presented along with the corresponding experimental data. These mean velocity profiles are non-dimensionalised by the use of $\overline{u_{m}}$ from Eq. 3 .

As mentioned earlier, the experimental relations are for the fully developed region of the jet flow.
Hence, further away from the jet exit (larger $\mathrm{x} / \mathrm{D}$ values), the numerical results better match the experimental data.

\section{Validation of the Stochastic Model Used:}

To check the accuracy of the turbulence field generated using CFWN model, we computed the temporal power spectral density of the fluctuating velocity at the center of the jet. The ensemble average of the computed power at each frequency is plotted with respect to the frequency and presented in Fig. 6. The slope of the computed averaged spectrum is compared to the line with $-5 / 3$ slope.

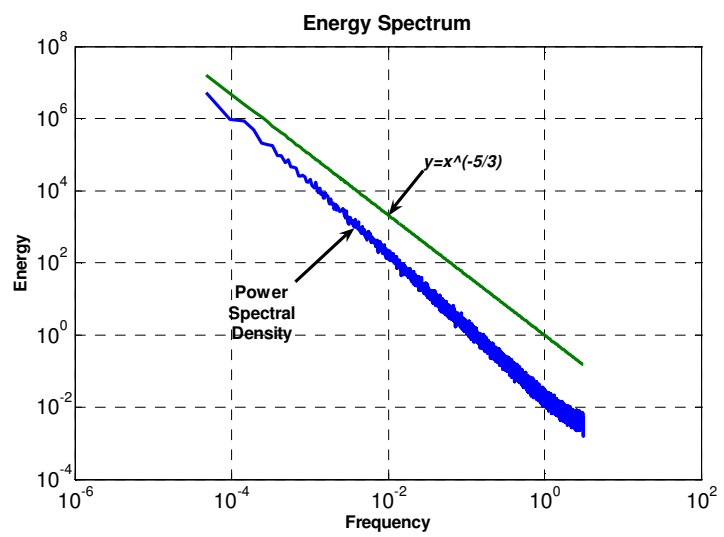

Fig. 6: Comparison of the computed power spectral density with the $-5 / 3$ slope line

As shown in Fig. 4, there is a region right after the jet outlet that has the same velocity as the jet exit. This region is called the potential core of the jet and has a wedge shape. In this region, the momentum of the still medium surrounding the jet has not diffused to reach the line of symmetry yet. This potential core can be observed in the velocity fluctuation contours of Fig. 7. Inside the core region, the flow is not turbulent and therefore no fluctuations are present.

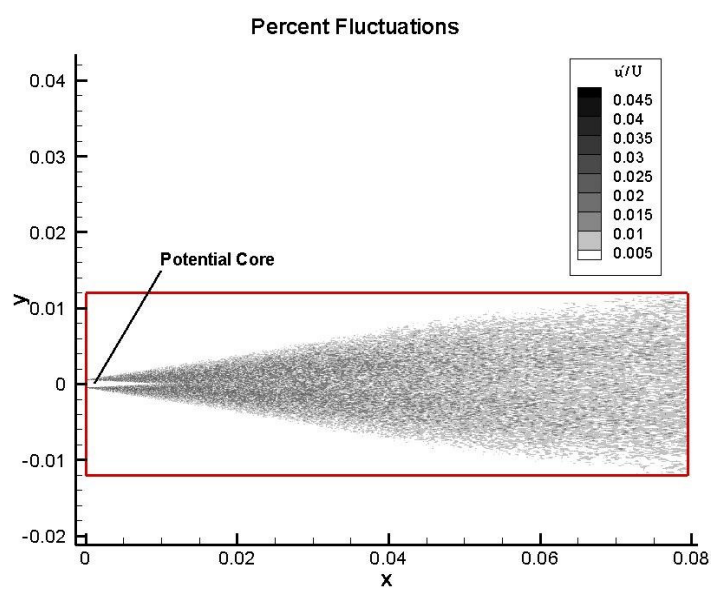

Fig.7: Velocity fluctuation contour showing no fluctuation in the core region of the jet 
It is known that the velocities at adjacent points in a turbulent flow are correlated to each other. These dependencies are expressed mathematically with the definition of two point spatial correlation. The CFWN method is categorized as a one point method, due to the fact that the computation for velocity fluctuations in one point does not affect the velocity fluctuations of its adjacent points. Hence, correct two-point spatial correlations can not be reproduced by this method.

\section{Results and Validation of the Far Field Noise:}

Power spectra of the density fluctuation at a distance of $\bar{X}=1000 \mathrm{D}$ (D is the jet exit width) from the nozzle exit is presented in Fig. 8.

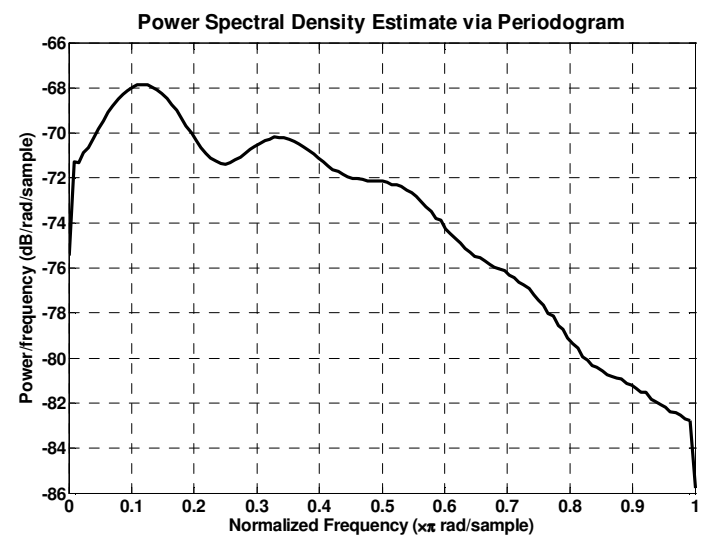

Fig. 8: Power spectra of the computed density fluctuations at a point $1000 \mathrm{D}$ from the jet

Since we evaluate the exact form of the Lighthill's volume integral, therefore it is possible to compute the contribution of the noise produced by any segment of the flow field separately. Different possible integration zones selected in this study to evaluate the volume integral are shown in Fig. 9.
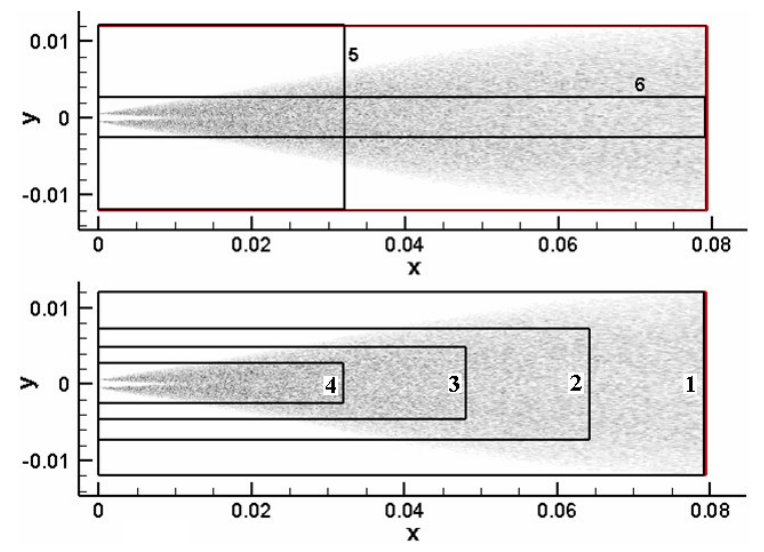

Fig. 9: Different possible integration zones of the flow field
Far from the source region of the jet where the acoustic fluctuations are governed by the linear wave equation, density and pressure fluctuations are related to each other as $p^{\prime}=c_{0}^{2} \rho^{\prime}$, so we can easily compute the magnitude of the pressure fluctuations, using the computed density fluctuations values ${ }^{[15]}$.

In Fig. 10, the overall sound pressure level, OSPL, as defined by Eq. 6, are shown for different integration regions of Fig. 9 on a half circle of radius $\bar{X}=200 \mathrm{D}$.

$O S P L=20 \log \left(\frac{p_{r m s}^{\prime}}{p_{r e f}}\right)$ where $p_{\text {ref }}=2 \times 10^{-5} \mathrm{~Pa}$

Overall Sound Pressure Level

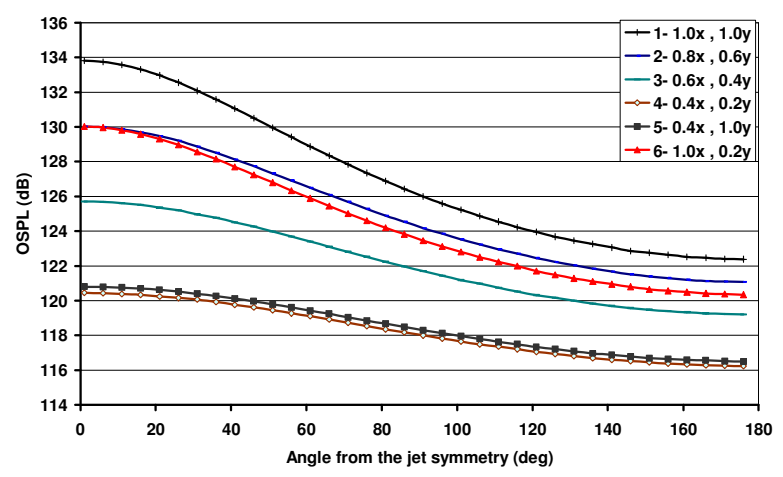

Fig.10: OSPL at 200D from the jet exit

By comparing different choices of integration zones and their corresponding OSPL, we can see that regions containing large velocity fluctuations are most effective in propagating sound to the far field. For example regions 4 and 5 that have the same length with different width, almost produce the same amount of sound. Even though zone 5 is much larger than zone 4, however they both contain almost the same amount of velocity fluctuations in them. Therefore, it is only necessary to integrate over the highly turbulent regions to compute the sound produced in jet flow.

\section{Overall Sound Pressure Level at Different Angles}

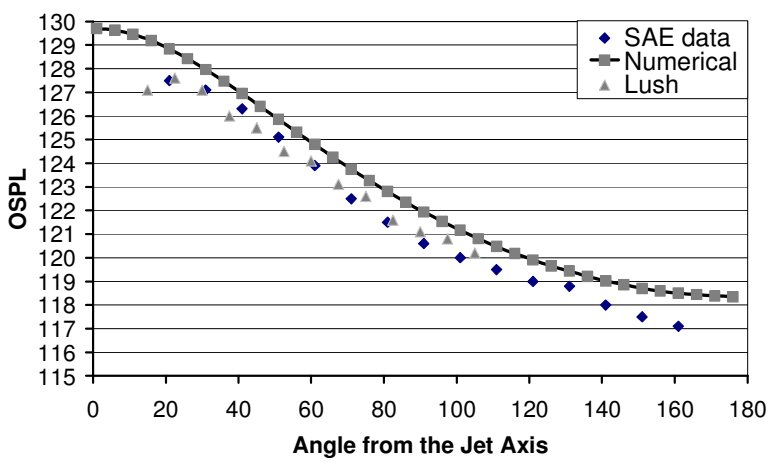

Fig.11: Comparison of the numerical results with the experimental data of $\mathrm{Lush}^{[16]}$ and $\mathrm{SAE}^{[17]}$ for $\mathrm{M}=0.56$ and $|\mathrm{X}|=120 \mathrm{D}$ 
In Fig. 11 the overall sound pressure levels from our numerical results are compared with the experimental data of $\operatorname{Lush}^{[16]}$ and SAE ${ }^{[17]}$. The OSPL on a half circle with the radius of 120D from the jet exit are presented. As shown in Fig. 11, the general trend in the numerical results is in reasonable agreement with the experimental data. The major difference between numerical results and experimental data is on predicting the maximum directivity angle of the jet. Numerical results show that the maximum directivity is at the jet axis ( 0 degree). There is no experimental data in vicinity of the jet axis because of the practical difficulties but it is known that the maximum directivity of the jet occurs at about 30 degrees from the jet axis.

There can be several reasons for this discrepancy. The CFWN method used here does not account for spatial structures that exist in real turbulent. As we know the directivity of the sound emitted from turbulent flows is due to large eddy structures existing in the flow. Hence, the discrepancy in the prediction of the maximum directivity can be expected. Also the size of the integral domain have to be large enough to contain all of the noise sources available in the flow, but as the CFD domain would become excessively large for far field calculations only a fraction of domain is considered here.

\section{CONCLUSIONS}

The stochastic method used here to simulate the velocity fluctuations satisfies the temporal properties of the turbulence. It also takes into account the intensity of turbulence flow. The calculated OSPL values and trends are in good agreement with the experimental data in the literature.

It seems that the combination of the CFWN method and Lighthill's volume integration is a good method for quick estimation of the OSPL with both reasonable computational speed and relatively good agreement with the experimental data.

This method is not as accurate as LES or DNS methods, but as the LES or DNS data at the near field are not always available or too costly to generate for most geometries, this method is a good alternative for finding quick estimates. This method is not limited to free jet problem and can be used in other geometries.

\section{REFERENCES}

1. Dowling, A.P, T. P. Hynes, 2004, sound generation by turbulence, European Journal of Mechanics B/Fluids, 23, 491-500

2. Lighthill, MJ., 1952, On sound generated aerodynamically: I. General theory., Proc. R.Soc. London, Ser. A, 211(1107), 564-587.
3. Bailly, C., Lafon P., and Candel S., 1997, Subsonic and supersonic jet noise predictions from statistical source models, AIAA journal, 35(11), 1688-1696.

4. Lyrintzis, A.S., Mankbadi R.R., 1996, Prediction of the Far-Field Jet Noise Using Kirchhoff's Formulation, AIAA J., 34(2), 413-416

5. Thomson, D. J., 1987, Criteria for the selection of stochastic models of particle trajectories in turbulent flow, J. Fluid Mech., Vol. 180, 529-556

6. Bodony, D.J, Lele S.K., 2003, A Statistical Subgrid Scale Noise Model: Formulation, 9th AIAA/CEAS Aeroacoustics Conference and Exhibit 12-14 May 2003, Hilton Head, South Carolina, 2003-3252

7. Morris, P.J, Farassat F., 2002, Acoustic Analogy and Alternative Theories for Jet Noise Prediction, AIAA J., Vol. 40, No. 4, 671-680

8. Wang, M., Freund J.B., and Lele S.K., 2006, Computational Prediction of Flow-Generated Sound, Annual Rev. Fluid Mech., Vol. 38, 483512

9. Perez, G., Prieur J., Rahier G. and Vuillot F., 2007, Theoretical and Numerical Discussion on Volume Integral Methods for Jet Noise Prediction, 13th AIAA/CEAS Aeroacoustics Conference, 20073593

10. He, C., Ahmadi G., 1999, Particle deposition in a nearly developed turbulent duct flow with electrophoreses, J. Aerosol Sci. Vol. 30, No. 6: 739-758

11. Box, G.E.P, and Muller M.E. ,1958, A note on the generation of random normal deviates, Annals Math. Stat, V. 29: 610-611.

12. Lele, S.K., 1992, Compact finite difference schemes with spectral-like resolution. J. Comp. Phys., Vol. 103:16-42

13. Zijnen, B.G., Hagge V., 1958, Measurements of the Velocity Distribution In a Plane Turbulent Jet of Air. App. Sci. Res., Vol. 7, Sect A

14. Görtler, H. Berechnung, 1942, Von Aufgaban der Freien Turbulnz auf Grund Eines Neunen Naherun Gsansatzes, Z.A.A.M, Vol. 22

15. Hirschberg, A., Rienstra S.W., 2007, Large-Eddy Simulation for Acoustics : Cambridge University Press, pp. 10-43

16. Lush, P. A., 1971, Measurements of Subsonic Jet Noise and Comparison with Theory, Journal of Fluid Mechanics, Vol. 46, No. 3: 477-500.

17. Society of Automotive Engineers, 1985, Gas Turbine Exhaust Noise Prediction, ARP 876C, Warrendale, PA 